\title{
AN EXPONENTIAL LIMIT FORMULA FOR NONLINEAR SEMIGROUPS
}

BY

\author{
JOEL L. MERMIN
}

\begin{abstract}
In recent papers, many writers have developed the theory of semigroups of operators generated by nonlinear accretive operators. In the present paper, we construct this semigroup by means of an exponential limit formula, and then use this means of obtaining the semigroup to prove an approximation theorem that is a direct generalization of the Kato-Trotter theorem for linear semigroups.
\end{abstract}

1. Introduction. In papers by Browder [1], Kato [3], and Komura [4], a theory of nonlinear semigroups has been developed in which a relatively simple sufficient condition that an operator $A$ generate such a semigroup, namely that $A$ be $m$ accretive (see $\S 2$ ), has been established. Once the existence of a semigroup having $A$ as its generator is known, it follows from results of Neuberger [6], and Ôharu [7] that the semigroup is given by the exponential limit formula $\lim _{n \rightarrow \infty}(I+t A / n)^{-n}$. In the main theorem of this paper presented in $\$ 3$, we show that this procedure can be reversed by proving directly that the above exponential limit is convergent for each $t \geqq 0$ to an operator $T_{t}$ if $A$ is $m$-accretive, and the family of operators so defined is a semigroup having $A$ as its generator. This result is close to some recent work of G. Webb [9] in which the semigroup generated by an $m$-accretive operator is defined by a more general product limit formula. It should be noted that the property we call " $m$-accretive" has been also called "hypermaximal accretive," and "hyper-accretive" by various authors.

In $\S 4$, we use the exponential limit formula to prove an approximation theorem for nonlinear semigroups which generalizes a linear theorem of Kato.

The author wishes to acknowledge his great indebtedness to Professor T. Kato who suggested the basic line of approach in the present work.

2. Duality mappings and $m$-accretive operators. Let $X$ be a Banach space, and $X^{*}$ its dual space. For each $x \in X$ and $f \in X^{*}$ denote $f(x)$ by $(x, f)$. A duality mapping on $X$ is a mapping $F: X \rightarrow X^{*}$ such that $(x, F(x))=|x|^{2}=|F(x)|^{2}$ for all $x$. In general, there will be many duality maps on $X$. If $X^{*}$ is uniformly convex however, then there is exactly one duality mapping $F$ and it is uniformly continuous on bounded subsets of $X$ (see [3]). Thus if $X^{*}$ is uniformly convex, we can define

Received by the editors August 19, 1969 and, in revised form, November 10, 1969.

AMS Subject Classfications. Primary 4750, 4780; Secondary 3495.

Key Words and Phrases. Nonlinear semigroups, accretive operators, exponential limit formula, nonlinear Hille-Yosida theorem, nonlinear semigroup approximation theorem.

Copyright (C) 1970, American Mathematical Society 
the function $\phi(R, \varepsilon)=\operatorname{lub}\{\delta \leqq 2 R|| x-y \mid<\delta$ and $|x| \leqq R$ implies $|F(x)-F(y)|<\varepsilon\}$ for all $R, \varepsilon>0$. Then $\phi$ is decreasing in $R$, increasing in $\varepsilon$, and

(2.1) $|x-y|<\phi(R, \varepsilon)$ and $|x| \leqq R$ implies $|F(x)-F(y)|<\varepsilon$.

From now on, we assume $X^{*}$ is uniformly convex.

An operator $A$ with domain and range in $X$ is accretive if and only if (2.2) $\operatorname{Re}(A x-A y, F(x-y)) \geqq 0$ for all $x, y \in D(A)$ for some duality map $F$.

$A$ is $m$-accretive if $A$ is accretive and

(2.3) $R(I+A)=X$.

It is easy to verify that $A$ accretive implies

(2.4) $(I+\lambda A)^{-1}$ is defined on $X$ and

$$
\left|(I+\lambda A)^{-1}\right|_{L} \leqq 1 \text { for all } \lambda>0
$$

where " ||$_{L}$ " is the Lipschitz norm of an operator. If $A$ is $m$-accretive it also follows that

(2.5) $R(I+\lambda A)=X$ for all $\lambda>0$ (see [3], [4], [5], [7]).

For $A m$-accretive, we set $J_{\lambda}=(I+\lambda A)^{-1}$, and $A_{\lambda}=A J_{\lambda}$. It follows that (2.6) $A_{\lambda}=\left(I-J_{\lambda}\right) / \lambda$.

LEMMA 2.1. (a) If $A$ is $m$-accretive, then for $x \in D(A)$

$$
\left|A J_{\lambda}^{k} x\right| \leqq|A x|, \quad k=1,2,3, \ldots, \lambda>0 .
$$

(b) If $A$ is m-accretive, and $x \in D(A)$, then

$$
\left|J_{\lambda_{1}}^{k} x-J_{\lambda_{2}}^{k} x\right| \leqq k \cdot|A x| \cdot\left|\lambda_{1}-\lambda_{2}\right|, \quad k=1,2,3, \ldots
$$

Proof. For $x \in D(A)$,

$$
\begin{aligned}
\left|A J_{\lambda}^{k} x\right| & =\left|A_{\lambda} \cdot J_{\lambda}^{k-1} x\right|=\frac{1}{\lambda}\left|J_{\lambda}^{k-1} x-J_{\lambda}^{k} x\right| \quad \text { (by (2.6)) } \\
& =\frac{1}{\lambda}\left|J_{\lambda}^{k} \cdot(I+\lambda A) x-J_{\lambda}^{k} x\right| \\
& \leqq \frac{1}{\lambda}|(I+\lambda A) x-x|=|A x| \quad \text { (by (2.4)). }
\end{aligned}
$$

Turning to (2.8), let $k=1$. If $u=J_{\lambda_{1} x}, v=J_{\lambda_{2} x}$, then $u+\lambda_{1} A u=v+\lambda_{2} A v$ and

$$
\begin{aligned}
|u-v|^{2}= & \left(\lambda_{2} A v-\lambda_{1} A u, F(u-v)\right)=\left(\lambda_{2}-\lambda_{1}\right) \operatorname{Re}(A v, F(u-v)) \\
& -\lambda_{1} \operatorname{Re}(A u-A v, F(u-v)) \leqq\left(\lambda_{2}-\lambda_{1}\right) \cdot \operatorname{Re}(A v, F(u-v)) \\
\leqq & \left|\lambda_{2}-\lambda_{1}\right| \cdot\left|A J_{\lambda_{2}} x\right| \cdot|u-v| .
\end{aligned}
$$

In view of (2.7), this proves (2.8) for $k=1$. One can now establish (2.8) for $k \geqq 1$ by an induction argument using the fact that $\left|J_{\lambda}\right|_{L} \leqq 1$.

If $A$ is $m$-accretive, then the following continuity property holds for $A$ :

(2.9) If $x_{n} \in D(A), \lim _{n \rightarrow \infty} x_{n}=x$, and $\left|A x_{n}\right| \leqq M$ for $n=1,2,3, \ldots$, then $x \in D(A)$ and wk $\lim _{n \rightarrow \infty} A x_{n}=A x$. (By wk $\lim _{n \rightarrow \infty} u_{n}=u$, we mean $\left(u_{n}, f\right)$ converges to 
$(u, f)$ for all $f \in X^{*}$.) Finally we need the following differentiation rule. If $|u(t)|$ and $u(t)$ are differentiable at $t_{0}$ and defined in an open interval about $t_{0}$, then

$$
(d / d t)|u(t)|_{t=t_{0}}^{2}=2 \operatorname{Re}\left(u^{\prime}\left(t_{0}\right), F u\left(t_{0}\right)\right) .
$$

For the proof of (2.9) and (2.10), see [3].

3. The exponential limit formula. A (nonlinear) semigroup on a set $D \subseteq X$ is a family of operators $T_{t}, t \geqq 0$ on $D$ such that:

1. $D\left(T_{t}\right)=D$ for all $t \geqq 0$,

2. $T_{0}=I_{I_{D}}$,

3. $T_{s+t}=T_{s} \cdot T_{t}$,

4. $T_{t} x$ is strongly continuous in $t$ for each $x \in D$,

5. $\left|T_{t}\right|_{L} \leqq 1$.

We note that many authors include only conditions 1-3 above in the definition of a semigroup, and use "strongly continuous contraction semigroup" for an object satisfying conditions 4 and 5 as well. Given a semigroup of operators, the operator $A$ defined by $A x=-$ wk $\lim _{h \rightarrow 0}\left(T_{h} x-x\right) / h$ with domain all $x$ for which this limit exists, is the weak infinitesimal generator of the semigroup.

If we replace weak limit by strong limit in the above, the resulting operator is the strong generator of the semigroup.

THEOREM 3.1. If $X^{*}$ is uniformly convex and $A$ is $m$-accretive on $X$, then for $x \in \mathrm{Cl}(D(A))$

$$
\lim _{n \rightarrow \infty}(I+t A / n)^{-n} x=T_{t} x
$$

exists for $t \geqq 0$, and the family of limit operators $T_{t}$ is a semigroup on $\mathrm{Cl}(D(A))$ having $A$ as its weak generator. If $X$ is uniformly convex as well, $A$ is the strong generator of the semigroup. Moreover, for each $a \in D(A), u(t)=T_{t} a$ is an almost everywhere strongly differentiable solution of

(3.1) $u^{\prime}(t)=-A u(t), u(0)=a$.

Proof. We begin by constructing a sequence of approximate solutions to (3.1). For $n=1,2,3, \ldots$, let $J_{n}=(I+A / n)^{-1}$. By $(2.4), D\left(J_{n}\right)=X$ and $\left|J_{n}\right|_{L} \leqq 1$ for all $n$. By (2.6) and (2.7)

(3.2) $A J_{n}=n\left(I-J_{n}\right)$,

(3.3) $\left|A J_{n}^{k} x\right| \leqq|A x|$ for $x \in D(A)$ and $n, k=1,2,3, \ldots$

For each $a \in D$, let

$u_{n}(t)=\left[J_{n}^{k-1}+n\left(t-\frac{k-1}{n}\right)\left(J_{n}^{k}-J_{n}^{k-1}\right)\right] a \quad$ for $\frac{k-1}{n} \leqq t \leqq \frac{k}{n}, \quad k=1,2,3, \ldots$

Then each $u_{n}$ is piecewise linear with $u_{n}(k / n)=J_{n}^{k} a$. In particular $u_{n}(0)=a$ for all $n$. If $(k-1) / n<t<k / n$, then

(3.4) $u_{n}^{\prime}(t)=n\left(J_{n}^{k}-J_{n}^{k-1}\right) a=-n\left(I-J_{n}\right) J_{n}^{k-1} a=-A J_{n}^{k} a=-u_{n}(k / n)$. 
It follows from (3.3) and (3.4) that

(3.5) $\left|u_{n}^{\prime}(t)\right| \leqq|A a|$ for almost all $t$, and all $n$.

Now let $x_{n m}(t)=u_{n}(t)-u_{m}(t)$ for $n, m \geqq 1$. Then $x_{n m}(0)=0$ and by (3.5)

(3.6) $\left|x_{n m}(t)\right| \leqq 2|A a| t$ for all $n, m$ and $t \geqq 0$.

Now each $\left|x_{n m}(t)\right|$ is Lipschitz continuous as $x_{n m}(t)$ is, so for almost all $t,\left|x_{n m}(t)\right|$ and $x_{n m}(t)$ are differentiable. Thus by (2.10) if $t$ satisfies $\left(k_{n}-1\right) / n<t<k_{n} / n$ for all $n$, $k_{n}$ a nonnegative integer, then for almost all $t$

$(d / d t)\left|x_{n m}(t)\right|^{2}=2 \operatorname{Re}\left(u_{n}^{\prime}(t)-u_{m}^{\prime}(t), \quad F x_{n m}(t)\right)=-2 \operatorname{Re}\left(A J_{n}^{k_{n}} a-A J_{m}^{k_{m}} a, F x_{n m}(t)\right)$.

Letting $y_{n m}(t)=J_{n}^{k_{n}} a-J_{m}^{k_{m}} a$, and using the accretiveness of $A$, the last term is dominated by

$$
2 \operatorname{Re}\left(A J_{n}^{k_{n}} a-A J_{m}^{k_{m}} a, F y_{n m}(t)-F x_{n m}(t)\right) \leqq 4|A a|\left|F y_{n m}(t)-F x_{n m}(t)\right|
$$

by (3.3). Hence $(d / d t)\left|x_{n m}(t)\right|^{2}<\delta$ almost everywhere in $[0, T]$ if

$$
\left|y_{n m}(t)-x_{n m}(t)\right|<\phi(2 T|A a|, \delta / 4|A a|)
$$

by (2.1) and (3.6). Since

$$
\left|y_{n m}(t)-x_{n m}(t)\right| \leqq\left|u_{n}(t)-u_{n}\left(\frac{k_{n}}{n}\right)\right|+\left|u_{m}(t)-u_{m}\left(\frac{k_{m}}{m}\right)\right| \leqq\left(\frac{1}{n}+\frac{1}{m}\right)|A a|,
$$

this will hold if

$$
n, m>\frac{2|A a|}{\phi(2 T|A a|, \delta / 4|A a|)} .
$$

Integrating and setting $\delta=\varepsilon^{2} / T$, we obtain

(3.7) $\left|x_{n m}(t)\right|=\left|u_{n}(t)-u_{m}(t)\right|<\varepsilon$ on $[0, T]$ if

$$
n, m>\frac{2|A a|}{\phi\left(2 T|A a|, \varepsilon^{2} / 4 T|A a|\right)} \text {. }
$$

Thus $u_{n}(t)$ converges uniformly on each bounded interval to a continuous $u(t)$ on $[0,+\infty)$. For any $t$, if $\left(k_{n}-1\right) / n \leqq t \leqq k_{n} / n$, then $\lim _{n \rightarrow \infty} k_{n} / n=t$ and by uniform convergence $u(t)=\lim _{n \rightarrow \infty} u_{n}\left(k_{n} / n\right)$ and $\left|A u_{i}\left(k_{n} / n\right)\right|=\left|A J_{n}^{k_{n}} a\right| \leqq|A a|$. Thus by (2.9) $u(t) \in D(A)$ and wk $\lim _{n \rightarrow \infty} A J_{n}^{k_{n}} a=A u(t)$. It follows that $|A u(t)| \leqq|A a|$ for all $t$, so that by (2.9) $A u(t)$ is weakly continuous, and for almost all $t$, wk $\lim _{n \rightarrow \infty} u_{n}^{\prime}(t)$ $=-A u(t)$. We have for any $f \in X^{*}$

$$
\left(u_{n}(t), f\right)=(a, f)+\int_{0}^{t}\left(u_{n}^{\prime}(s), f\right) d s
$$

where the integrand converges boundedly almost everywhere to $-A u(s)$. Thus letting $n \rightarrow \infty$ we get

$$
(u(t), f)=(a, f)-\int_{0}^{t}(A u(s), f) d s .
$$

Since the integrand is continuous, $u(t)$ has the weakly continuous weak derivative $-A u(t)$ everywhere in $[0,+\infty)$. Since weak continuity implies $A u(s)$ is Bochner 
integrable, we get $u(t)=a-\int_{0}^{t} A u(s) d s$ for all $t$ and $u(t)$ has $-A u(t)$ has a strong derivative almost everywhere by a standard result about Bochner integrals (see [8]).

Now let $v_{n}(t)=(I+t A / n) a^{-n}$ for $a \in D(A)$. Note that $v_{n}(1)=u_{n}(1)$ for all $n$. Applying the convergence estimate (3.7) with $t=T=1$, but to $t A$ in place of $A$ we get

$$
\left|v_{n}(t)-v_{m}(t)\right|<\varepsilon \text { for } n, m>\frac{2 t|A a|}{\phi\left(2 t|A a|, \varepsilon^{2} / 4 t|A a|\right)} .
$$

It follows that $v_{n}(t)$ is uniformly convergent on any bounded interval to a limit $v(t)$. Since for $t=p / q$ rational $u_{q n}(p / q)=v_{p n}(p / q)$, in the limit $v(t)=u(t)$ for $t$ rational. But the $v_{n}(t)$ are uniformly continuous by (2.8) which implies

$$
\left|v_{n}\left(t_{1}\right)-v_{n}\left(t_{2}\right)\right| \leqq|A a| \cdot\left|t_{1}-t_{2}\right| \text {. }
$$

Thus $v$ is continuous and $v(t)=u(t)$ for all $t$. So for any

$$
a \in D(A) \lim _{n \rightarrow \infty}(I+t A / n)^{-n} a=u(t)
$$

where $u(t)$ is a solution of (3.1). Since $\left|(I+t A / n)^{-n}\right|_{L} \leqq 1$, we easily obtain that $\lim _{n \rightarrow \infty}(I+t A / n)^{-n} x=T_{t} x$ exists for any $x \in \mathrm{Cl}(D(A))$. Clearly $\left|T_{t}\right|_{L} \leqq 1$, and this together with the continuity of $T_{t} a$ for $a \in D(A)$ gives continuity of $T_{t} x$ for all $x \in \mathrm{Cl}(D(A))$.

Also $T_{0}=I$ on $D$, and for any $t \geqq 0$ and positive integer $m$

$$
T_{t m}=\lim _{n \rightarrow \infty}\left(I+\frac{t m}{n} A\right)^{-n}=\lim _{n \rightarrow \infty}\left(I+\frac{t}{n} A\right)^{-n m}=T_{t}^{m}
$$

It is easy to deduce from this that $T_{s+t}=T_{s} \cdot T_{t}$.

Thus $T_{t}, t \geqq 0$ is a semigroup, and we have left to show that $A$ is its generator. For $a \in D(A), T_{t} a=u(t)$ satisfies (3.1) weakly at 0 so that

$$
-A a=\text { wk } \lim _{h \rightarrow 0}\left(T_{h} a-a\right) / h \text {. }
$$

Thus $A \subseteq \hat{A}$ where $\hat{A}$ is the weak generator of $A$. But $\hat{A}$ is accretive (see [3]) so that $I+\hat{A}$ is a one-to-one extension of $I+A$ which has range $X$. This is possible only if $A=\hat{A}$.

Finally, suppose $X$ is uniformly convex. To show $A$ is the strong generator, we must show $-A a=\lim _{h \rightarrow 0}\left(T_{h} a-a\right) / h$. But

$$
\frac{T_{h} a-a}{h}=\frac{-1}{h} \int_{0}^{h}\left(A T_{s} a\right) d s
$$

using a Bochner integral. Hence it suffices to show that $\lim _{h \rightarrow 0} A T_{h} a=A a$. This limit holds weakly so that $|A a| \leqq \lim _{h \rightarrow 0}$ inf $\left|A T_{h} a\right|$ but $\left|A T_{h} a\right|=|A u(h)| \leqq|A a|$ for $h>0$

$$
\therefore \lim _{h \rightarrow 0}\left|A T_{h} a\right|=|A a|
$$

and so by uniform convexity of $X \lim _{h \rightarrow 0} A T_{h} a=A a$. 


\section{An approximation theorem.}

TheOREM 4.1. Let $A, A_{k}, k=1,2,3, \ldots$, be m-accretive operators on $X$ where $X^{*}$ is uniformly convex. Let $T_{t}$, and $T_{t}^{k}, k=1,2,3, \ldots$, be the associated semigroups. Suppose that

1. $\mathrm{Cl}\left(D\left(A_{k}\right)\right)=\mathrm{Cl}(D(A)), k=1,2,3, \ldots$

2. $\lim _{k \rightarrow \infty}\left(I+\lambda_{0} A_{k}\right)^{-1} x=\left(I+\lambda_{0} A\right)^{-1} x$, for some $\lambda_{0}>0$ and all $x \in X$.

Then for all $x \in \mathrm{Cl}(D(A)), \lim _{k \rightarrow \infty} T_{t}^{k} x=T_{t} x$, the limit being uniform on bounded $t$ intervals for each $x$ in $D(A)$.

We need two preliminary lemmas.

LEMMA 4.1. Under the hypotheses of Theorem 4.1,

$$
\lim _{k \rightarrow \infty}\left(I+\lambda A_{k}\right)^{-n} x=(I+\lambda A)^{-n} x \text { for all } \lambda>0, x \in X \text { and } n=1,2,3, \ldots
$$

Proof. Let $n=1$, and set $R_{k}(\lambda)=\left(\lambda I+A_{k}\right)^{-1}, k=1,2,3, \ldots$, and $R(\lambda)=(\lambda I+A)^{-1}$. Since $R(\lambda)=\left(I+\lambda^{-1} A\right)^{-1} \cdot \lambda^{-1}, R_{k}(\lambda)=\left(I+\lambda^{-1} A_{k}\right)^{-1} \cdot \lambda^{-1}$, it is sufficient to show that $\lim _{k \rightarrow \infty} R_{k}(\lambda) x=R(\lambda) x$ holds for all $\lambda>0$ if it holds for a single $\lambda_{0}>0$. We show that if it holds for $\lambda_{0}>0$, then it holds for all $\lambda$ such that $\left|\lambda-\lambda_{0}\right|<\lambda_{0}$ from which the desired conclusion easily follows.

Suppose $\lim _{k \rightarrow \infty} R_{k}\left(\lambda_{0}\right) x=R\left(\lambda_{0}\right) x$ for all $x \in X$. Let $\lambda$ satisfy $\left|\lambda-\lambda_{0}\right|<\lambda_{0}$, and let $u_{k}=R_{k}(\lambda) x, u=R(\lambda) x$. Then $\lambda u_{k}+A_{k} u_{k}=x$, so that $\left(\lambda-\lambda_{0}\right) u_{k}+\left(\lambda_{0} I+A_{k}\right) u_{k}=x$ and $u_{k}=R_{k}\left(\lambda_{0}\right)\left(x-\left(\lambda-\lambda_{0}\right) u_{k}\right)$. Similarly, $u=R\left(\lambda_{0}\right)\left(x-\left(\lambda-\lambda_{0}\right) u\right)$. Then

$$
\begin{aligned}
\left|u-u_{k}\right| \leqq & \left|u-R_{k}\left(\lambda_{0}\right)\left(x-\left(\lambda-\lambda_{0}\right) u\right)\right| \\
& +\left|R_{k}\left(\lambda_{0}\right)\left(x-\left(\lambda-\lambda_{0}\right) u\right)-R_{k}\left(\lambda_{0}\right)\left(x-\lambda-\lambda_{0}\right) u_{k}\right| .
\end{aligned}
$$

By hypothesis, the first term on the right in (4.1) tends to 0 as $k \rightarrow \infty$, and the second term is dominated by

$$
\left|R_{k}\left(\lambda_{0}\right)\right|_{L} \cdot\left|\lambda-\lambda_{0}\right| \cdot\left|u-u_{k}\right| \leqq\left|\lambda-\lambda_{0}\right| /\left|\lambda_{0}\right| \cdot\left|u-u_{k}\right| \cdot
$$

Setting $c=\left|\lambda-\lambda_{0}\right| /\left|\lambda_{0}\right|<1$, it follows that $\left|u-u_{k}\right|(1-c)$ is dominated by a sequence converging to 0 so

$$
\lim _{k \rightarrow \infty} u_{k}=\left(\lambda I+A_{k}\right)^{-1} x=u=(\lambda I+A)^{-1} x .
$$

The convergence can be extended to $n>1$, by induction using the uniform Lipschitz continuity of the operators $\left(\lambda I+A_{k}\right)^{-n}$.

LemMA 4.2. Let $x_{k} \in D\left(A_{k}\right), k=1,2,3, \ldots$, where $\lim _{k \rightarrow \infty} x_{k}=x$ and $\left|A_{k} x_{k}\right| \leqq M$ for all $k$ and some $M>0$. Then for any fixed $n, \lim _{k \rightarrow \infty}\left(I+t A_{k} / n\right)^{-n} x_{k}=(I+t A / n)^{-n} x$ the convergence being uniform on any bounded $t$ interval. 
Proof. The pointwise existence of the above limit follows from Lemma 4.1 since

$$
\begin{aligned}
\left|\left(I+\frac{t}{n} A_{k}\right)^{-n} x_{k}-\left(I+\frac{t}{n} A\right)^{-n} x\right| \leqq & \left|\left(I+\frac{t}{n} A_{k}\right)^{-n} x-\left(I+\frac{t}{n} A\right)^{-n} x\right| \\
& +\left|\left(I+\frac{t}{n} A_{k}\right)^{-n} x_{k}-\left(I+\frac{t}{n} A_{k}\right)^{n} x\right|
\end{aligned}
$$

where the first term tends to zero by Lemma 4.1 and the second term, dominated by $\left|x_{k}-x\right|$ also tends to 0 as $k \rightarrow \infty$. But in view of (2.8)

$$
\left|\left(I+\frac{t_{1}}{n} A_{k}\right)^{-n} x_{k}-\left(I+\frac{t_{2}}{n} A_{k}\right)^{-n} x_{k}\right| \leqq\left|t_{1}-t_{2}\right| \cdot\left|A_{k} x_{k}\right| \leqq\left|t_{1}-t_{2}\right| \cdot M .
$$

Thus the sequence $\left(I+t A_{k} / n\right)^{-n} x_{k}$ is equicontinuous in $t$ and the convergence must be uniform in bounded $t$ intervals.

We can now prove the theorem. Since the semigroup operators all have Lipschitz norm uniformly less than 1 it suffices to show $\lim _{k \rightarrow \infty} T_{t}^{k} x=T_{t} x$ uniformly for $0 \leqq t \leqq T$ for any $T>0$ for all $x$ in the dense set $D(A)$. For such an $x$, we have $x=(I+A)^{-1} u$ for some $u \in X$, and setting $x_{k}=\left(I+A_{k}\right)^{-1} u, k=1,2,3, \ldots$, we have $\lim _{k \rightarrow \infty} x_{k}=x$ and since $A_{k} x_{k}=u-x_{k}$, we also have $\left|A_{k} x_{k}\right| \leqq M$ for all $k$, and some $M>0$. Then

$$
\begin{aligned}
\left|T_{t}^{k} x-T_{t} x\right| \leqq & \left|T_{t}^{k} x-T_{t}^{k} x_{k}\right|+\left|T_{t}^{k} x_{k}-\left(I+\frac{t}{n} A_{k}\right)^{-n} x_{k}\right| \\
& +\left|\left(I+\frac{t}{n} A_{k}\right)^{-n} x_{k}-\left(I+\frac{t}{n} A\right)^{-n} x\right| \\
& +\left|\left(I+\frac{t}{n} A\right)^{-n} x-T_{t} x\right| .
\end{aligned}
$$

Let $\varepsilon>0$ be given and let $0 \leqq t \leqq T$. By (3.8) using the fact that $\left|A_{k} x_{k}\right| \leqq M$, the second and fourth terms of (4.2) can be made less than $\varepsilon / 4$ for all $n \geqq n_{0}$ independent of $k$. The first term of (4.2) is dominated by $\left|x-x_{k}\right|$ and so is less than $\varepsilon / 4$ for $k \geqq k_{0}$. Finally, taking $n=n_{0}$, he third term is less than $\varepsilon / 4$ for $k \geqq k_{1}$ by Lemma 4.2. Thus taking $n=n_{0}$, and $k>k_{0}, k_{1},\left|T_{t}^{k} x-T_{t} x\right|<\varepsilon$ for $0 \leqq t \leqq T$.

Finally, we remark that our two theorems can be generalized to the case where $A=A(t)$ is a family of $m$-accretive operators depending on $t$, for $t \geqq 0$. If the domain $D$ of $A(t)$ is independent of $t$, and $A(t)$ varies smoothly with $t$ in a certain sense, then the solutions of $u^{\prime}(t)=-A(t) u(t), u(0)=a$ can be obtained by the " time dependent" exponential formula

$$
\lim _{n \rightarrow \infty} \prod_{k \leqq n}\left(I+\frac{t}{n} A\left(\frac{k t}{n}\right)\right)^{-1} a=M(t) a .
$$

The $M(t), t \geqq 0$ are a family of evolution operators associated with $A(t)$, and our second theorem can be generalized as follows. If $A_{k}(t), k=1,2,3, \ldots$, and $A(t)$ 
are families of $m$-accretive operators with $D\left(A_{k}(t)\right)=D_{k}, k=1,2,3, \ldots, D(A(t))$ $=D$ independent of $t$, and $\bar{D}_{k}=\bar{D}=X$ for each $k$, where a certain common smoothness condition in $t$ is satisfied by all operator families, then if

$$
\lim _{k \rightarrow \infty}\left(I+\lambda(t) A_{k}(t)\right)^{-1}=\left(I+\lambda(t) A_{k}(t)\right)^{-1}
$$

for each $t \geqq 0$ and some $\lambda(t)>0$, then $\lim _{k \rightarrow \infty} M_{k}(t) x=M(t) x$ uniformly for $t$ in any bounded interval, and any $x \in X$, where $M_{k}(t), t \geqq 0$ is the family of evolution operators associated with $A_{k}(t), k=1,2,3, \ldots$ For these time dependent results, see [5].

\section{BIBLIOGRAPHY}

1. F. E. Browder, Nonlinear equations of evolution and nonlinear accretive operators in Banach spaces, Bull. Amer. Math. Soc. 73 (1967), 867-874. MR 38 \#580.

2. T. Kato, Perturbation theory for linear operators, Die Grundlehren der math. Wissenschaften, Band 132, Springer-Verlag, Berlin and New York, 1966. MR 34 \#3324.

3. - Nonlinear semigroups and evolution equations, J. Math. Soc. Japan 19 (1967), 508-520. MR 37 \#1820.

4. Y. Komura, Nonlinear semi-groups in Hilbert space, J. Math. Soc. Japan 19 (1967), 493-507. MR 37 \#7176.

5. J. Mermin, Accretive operators and nonlinear semigroups, Thesis, Univ. of California, Berkeley, 1968.

6. J. W. Neuberger, An exponential formula for one-parameter semi-groups, J. Math. Soc. Japan 18 (1966), 154-157. MR 34 \#622.

7. S. Ôharu, Note on the representation of semi-groups of non-linear operators, Proc. Japan Acad. 42 (1966), 1149-1154. MR 36 \#3167.

8. K. Yosida, Functional analysis, Die Grundlehren der math. Wissenschaften, Band 123, Academic Press, New York and Springer-Verlag, Berlin and New York, 1965. MR 31 \#5054.

9. G. Webb, Nonlinear evolution equations and product integrals, (to appear).

UNIVERSITY OF CONNECTICUT,

STORRS, CONNECTICUT 06268 